\title{
Aloe Vera Extracted Polysaccharides Shift the Immune Responses of Tumor Bearing Mice Toward Th1 Pattern: Animal Study
}

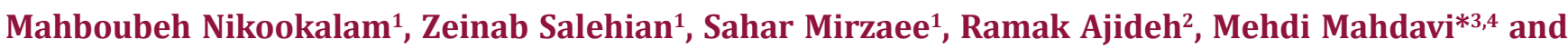 \\ Mohammad Hossein Yazdi*2,3,4
}

${ }^{1}$ Department of Biotechnology, Faculty of Advanced Sciences \& Technology, Tehran Medical Sciences, Islamic Azad University, Tehran-Iran (IAUPS)

${ }^{2}$ Biotechnology Research Center, Faculty of Pharmacy, Tehran University of Medical Sciences, Tehran, Iran

${ }^{3}$ Recombinant Vaccine Research Center, Tehran University of Medical Sciences, Tehran, Iran

${ }^{4}$ Immunotherapy Group, The Institute of Pharmaceutical Sciences (TIPS), Tehran University of Medical Sciences, Tehran, Iran

*Corresponding author: Mohammad Hossein Yazdi, Biotechnology Research Center and Recombinant Vaccine Research Center, Faculty of Pharmacy, Tehran University of Medical Sciences, Tehran, Iran. Immunotherapy Group, The Institute of Pharmaceutical Sciences (TIPS), Tehran University of Medical Sciences, Tehran, Iran

Mehdi Mahdavic, Recombinant Vaccine Research Center, Faculty of Pharmacy, Tehran University of Medical Sciences, Tehran, Iran

\section{ARTICLE INFO}

Received: 慧 March 03, 2019

Published: 㟽 March 29, 2019

Citation: Mahboubeh Nikookalam, Zeinab Salehian, Sahar Mirzaee, Ramak Ajideh, Mehdi Mahdavi, Mohammad HosseinY. Aloe Vera Extracted Polysaccharides Shift the Immune Responses of Tumor Bearing Mice Toward Th1 Pattern: Animal Study. Biomed J Sci \& Tech Res 16(4)-2019. BJSTR. MS.ID.002873.

Keywords: Aloe Vera; Glucomannan; Adjuvant; Immune response; Th1/Th2; Cancer

Abbreviations: TSA: Tumor Specific Antigen; FDA: America's Food and Drug Agency; TSA: Tumor Specific Antigen; CTL: Cytotoxic T Lymphocyte; Alum: Aluminum hydroxide; RTV: Relative Tumor Volume
ABSTRACT

Background and Aim: In this work, the effect of oral administration of Aloe Vera extracted polysaccharides were investigated on the immunization profile of TC1 induced tumor bearing mice with and without HPV16 E7d vaccine.

Experimental Procedure: Sixty C57BL/6 mice were divided into six groups as follow: Group 1, E7d-ALUM-GEL; Group 2, E7d-ALUM; Group 3, E7d-MONTANIDE ISA 206GEL; Group 4, E7d-MONTANIDE ISA 206; Group 5 and Group 6, GEL ALONE and PBS . The oral feeding of Aloe Vera gel with $0.05 \%$ polysaccharide was done for a period of 30 days. Mice were immunized with HPV16 E7d vaccine three times on day 0,14 and 28th. Then tumor was implanted subcoutaneosly and 2 weeks later, the serum samples of the mice were collected to evaluate IL- 4 and IFN- $\gamma$ cytokines and specific total IgG, IgG1 and IgG2a antibodies levels by ELISA.

Result: Immunization of mice with E7d-ALUM-GEL significantly increased the IFN- $\gamma$ and IL-4 immune response levels in comparison to other vaccinated groups. It is important to mentioned that gel alone group not only showed an increase in the ratio of IFN- $\gamma$ / IL-4 in comparison to other groups but also demonstrated the greatest reduction in proportion of tumor growth. Meanwhile, the best survival rate was recorded in E7d -ALUM-GEL and GEL ALONE groups.

Conclusion: Aloe Vera gel contains mannose containing plant compounds like Acemannan and Glucomannan which stimulate the immune responses and can be used as an adjuvant element or as supplement for cancer patients without serious concern about toxicity (Figure 1). 


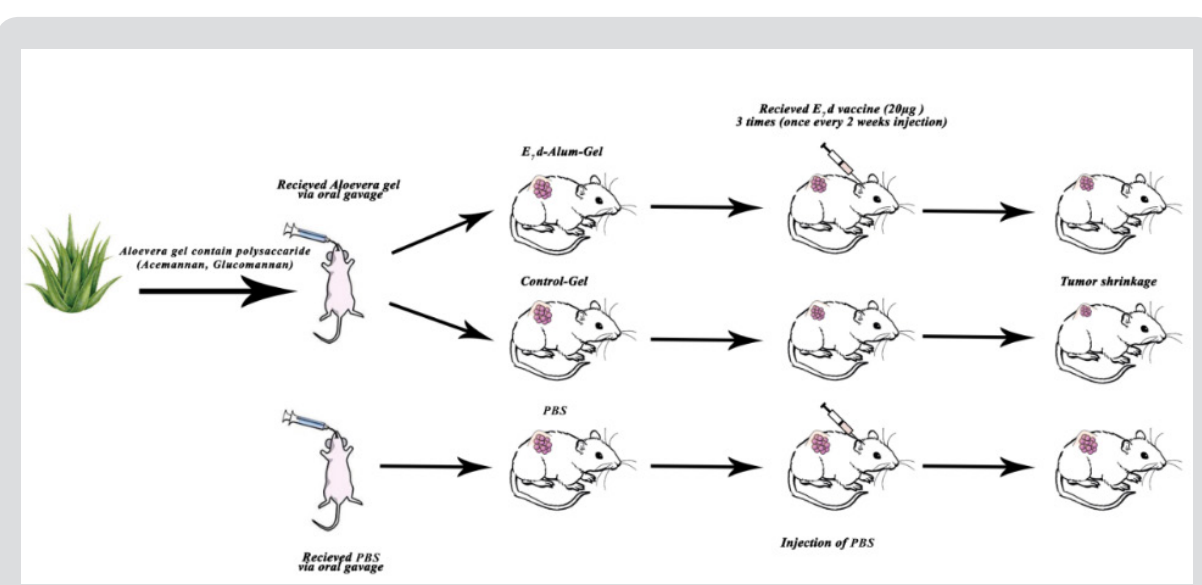

Figure 1.

\section{Introduction}

Cervical cancer is a public health problem and is well-known as the fourth most common cancer and the fourth major cause of cancer-associated death in women in the developing countries [1]. Human Papilloma Virus (HPV) may cause an infection with no symptom and would be self-alleviated but infection persistansy of some HPV species may cause warts and also precancerous lesions [2,3]. This virus, especially its "high-risk" genotypes such as HPV16 and 18 are shown to have a critical role as the most important etiological factor in the induction of cervical cancer [46]. The proteins E6 and E7 are two oncogenes that are necessary for malignant conversion and are expressed in oncogenic HPVs (e.g, types 16 and 18) and have transforming properties. To date, vaccination has been the most effective method to improve and control infectious diseases in terms of effectiveness and economy [7]. Prophylactic vaccines such as Gardasil and Cervarix which are approved by America's Food and Drug Agency (FDA), stimulate humoral immune response by induction of antibodies secreation against non-infectious Virus Like Particles (VLPs) which are part of Major capsid protein of HPV (L1 of HPV types 6, 11, 16, and 18 for Gardasil, and types 16, and 18 for Ceravix) [8,9]. HPV16E7 is a small protein (11KDa) known as Tumor Specific Antigen (TSA) has been expressed in eukaryotic and prokaryotic cells and agitated immune cells against, TC-1 tumor growth (TC-1 is the mouse tumor cell line, which expresses the E6 and E7 oncoprotein of HPV-16).

Recombinant E7 protein is a good candidate as a therapeutic HPV vaccine, but it is not enough immunogen for stimulating the cytotoxic T lymphocyte (CTL) responses [10-14]. Aluminum hydroxide (Alum) is the most common used adjuvant for human vaccines. Alum although is able to stimulate antibody secretion and shifts immune responses toward Th2, it has low potency for stimulating the cellular (Th1) immune responses which beside Th2 and humoral immunity is so important for protection antigens HPV infection. Montanide is another adjuvant that raises the TH1 response and improves IgG2a production [15-17]. In vitro and In vivo studies on herbal derivatives demonstrated that glucose and mannose-based carbohydrate polymers which are derived from plants (like Aloevara) and fungus are able to stimulate the reticulo-endothelial system and induce both Th1 and Th2 immune responses. For this reason and as these polysaccharide ingredients revealed not any local or systemic toxicity, we proposed to use Aloe Vera gel extracted polysaccharide as adjuvant. Studies showed that immunostimulating and antitumor activity effect of Aloe Vera plant are due to the existence of a polysaccharides named Acemannan and Glucomannan which are considered as major carbohydrate fractions, obtained from the gel of the Aloe Vera leaf [18-20]. It is recommended that the macrophage mannose receptor as a carbohydrate-binding endocytic receptor, can adjoin to Acemannan mannose residues [21,22]. Glocomannan has a great declination to coupling with macrophage that express mannose receptors on their surface abundantly. Meanwhile, Glucomannan is an inhibitor of the tumor-associated macrophages (TAMs) activity. TAMs play the main role in tumor angiogenesis and metastasis [23]. Glucomannan and Acemannan will trigger the mannose receptors on the surface of macrophages and thereby increase the expression of pro-inflammatory cytokines, nitric oxide and agitate phagocytosis $[14,24]$. In the present work, we hypothesized that the oral feeding of Aloe Vera gel containing natural polysaccharides to mice which are immunized with HPV16E7d by stimulating the immune responses may be result in improvement of $\mathrm{E} 7 \mathrm{~d}$ vaccine efficacy or treatment outcome.

\section{Materials and Methods}

\section{Experimental Animals}

Seven-weeks-old male C57BL/6 mice ( $\mathrm{n}=65$, 19.4-22.9gm) (Supp Figure) were purchased from the Pasteur Institute of Iran (Karaj, Iran) and were manipulated and maintained under standard 
conventional conditions (free access to food and water, in a light/ dark cycle with lights on from 6:00 a.m to 6:00 p.m) according to

the care and handling Protocol of Pasteur Institute of Iran for one week before the experiments.

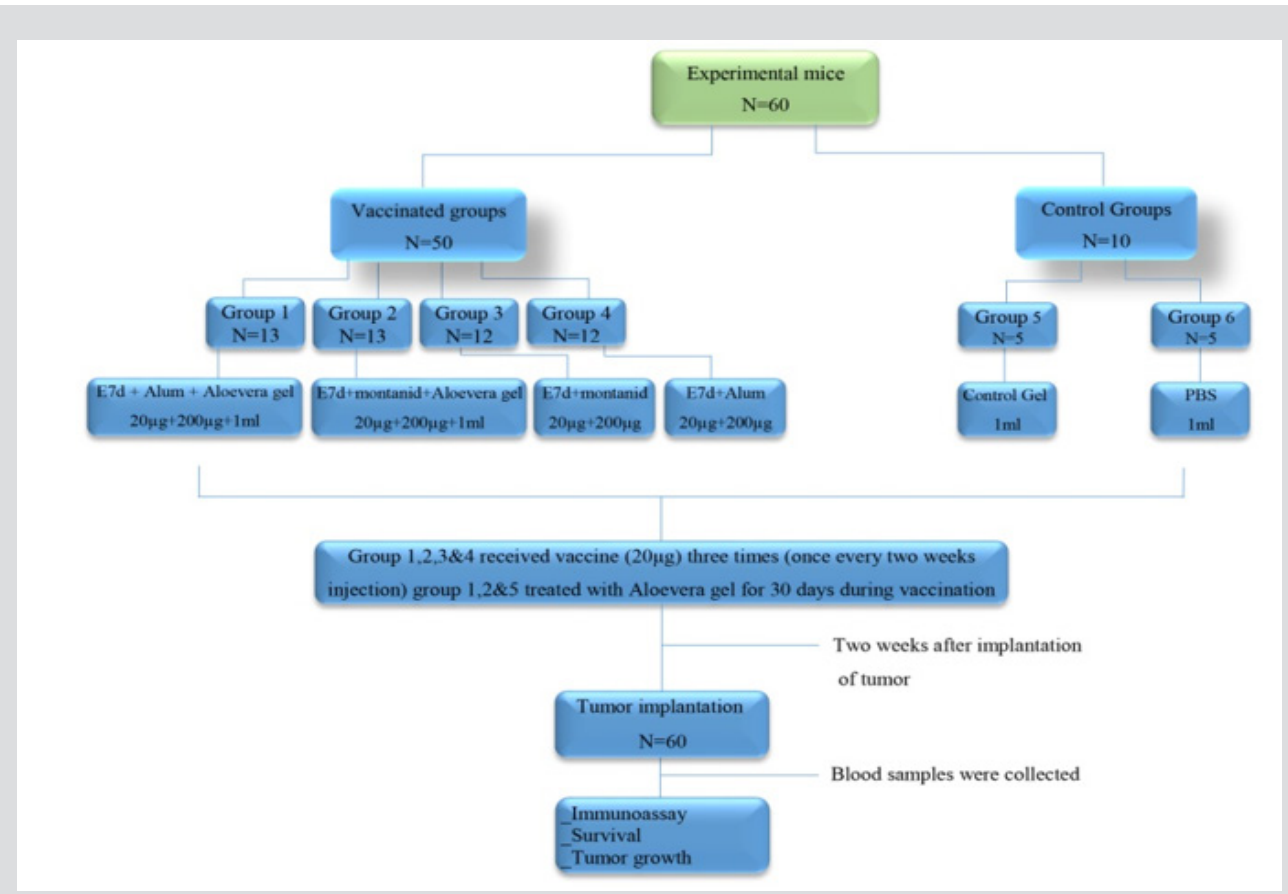

Supplementary Figure: The diagram of methods and planning of study.

\section{TC-1 Cell Culture}

The TC-1 cell line was prepared from Department of Cell Bank in Pasteur Institute of Iran and propagated in complete RPMI-1640 to a high density.

\section{Injection of TC-1 Cell Line to Prepare Stock Tumor Mice}

The mice that were injected with TC-1 cells line are called stock mice which were used as a source of tumor tissue for induction of tumor through surgery in pre-vaccinated mice. Cultivated TC-1 cell line were prepared in PBS buffer and brought the suspension to density of $1 \times 107$ cells $/ \mathrm{ml}$ and $100 \mu \mathrm{l}$ and injected into the right-side of five mice flank; and waiting for tumor growth for two or three weeks.

\section{Tumor Implantation}

Initially, Mice flank were shaved by depilatory, then mice were anesthetized by injection of $200 \mu \mathrm{l}$ of ketamine/xylazin combination (10\% ketamine HCL + 5\% xalyzin + PBS buffer) and were euthanized by spinal dislocation. Then the tumors were removed from the body of mice in sterile conditions and were divided into 3-5 mm pieces and preserved in cold PBS buffer containing penicillin and streptomycin antibiotics for transplantation under the skin of anesthetized experimental mice by surgery.

\section{Vaccine Formulation}

The HPV16 E7d vaccine was prepared in combination of two different adjuvant, aluminum hydroxide adjuvant (Alum, Pasteur Institute of Iran, Karaj, Iran) and Montanide ISA 206 adjuvant. For this purpose, the HPV-16 E7d vaccine was first dissolved in sterile PBS and then mixed with alum and/or Montanide ISA 206. Mice from group I and IV were vaccinated with E7d vaccine contained $20 \mu \mathrm{g}$ of HPV16 E7d antigen and $200 \mu \mathrm{g}$ of Alum, and group II and III were vaccinated with E7d vaccine contained 20 $\mathrm{g}$ of HPV16 E7d antigen formulated in Montanide ISA 206 adjuvant.

\section{Immunization and Study Planning}

The mice were divided into six groups containing: Group I, E7d adjuvanted in Alum and Aloe Vera gel oral feeding (E7d-Alum \& Aloe Vera gel, $n=13)$; Group II, E7d adjuvanted in Montanide ISA 206 and Aloe Vera Gel oral feeding (E7d-Montanide ISA 206 \& Aloe Vera Gel, n=13);Group III, E7d adjuvanted in Montanide ISA 206 (E7d-Montanide ISA 206, n=12); Group IV, E7d adjuvanted in alum (E7d-Alum,n=12); Group V, PBS oral feeding (as control group, $\mathrm{n}=$ 5); Group VI, Aloe Vera gel oral feeding (as GEL ALONE group, $n=5$ ). Mice from group I, II, III and IV were vaccinated three times with 10 days intervals.

\section{Oral Administration of Aloe Vera Gel During Vaccination}

The oral feeding of mice from group I, II and VI accomplished with Aloe Vera Gel (Barij Essence ,Iran) containing 0.05\% polysaccharide by daily oral gavage administration (1 $\mathrm{ml}$ of Aloe Vera Gel ) for a period of 30 days. After first five days of Aloe Vera Gel oral feeding of the mice from group I (E7d-Alum \& Aloe Vera gel) and II (E7d-Montanide ISA 206 \& Aloe Vera Gel), they were immunized by s.c injection of E7d vaccine in a total volume $100 \mu \mathrm{l}$. Vaccines injection were performed three times (Once every 10 days 
injections) via subcutaneous route for mice from group I, II, III and IV. Oral administration of Aloe Vera (for group I, II and VI) and PBS (for group V) were continued for 30 days.

\section{Serum Collection}

Two weeks after implantation of tumor in the experimental group, blood samples were collected from the inner corner of the eyes and blood serum was collected with centrifuge and saved at $-200 \mathrm{C}$ until use.

\section{Tumor Growth Evaluation}

In this study, the largest length and width of tumor were measured by Digital caliper. Also, the experimental mice tumor volume $\left(\mathrm{mm}^{3}\right)$ were measured by formula of $\mathrm{ab}^{2} / 2$. Relative Tumor Volume (RTV) increase can be calculated by dividing the last day tumor size to the first day of transplanted tissue and this index was used in the statistical calculations.

\section{Survival of Mice}

After induction of tumor, mice were kept in cages for 50 days and were daily recorded for the mortality trends due to tumor progression.

\section{Specific Total IgG and Antibody Assay}

Specific total IgG antibody was evaluated in the serum sample which was collected two weeks after transplantation of tumor tissue to assay specific humoral immune responses with an optimized indirect ELISA method.

\section{Cytokines Assay}

Cytokines (IFN- $\gamma$ and IL-4) assay was done on serum samples which was collected two weeks after the immunization by ELISA Kit (Mabtech, Sweden) according to the manufacturer's protocol.

\section{Statistical Analysis}

The data presented as means \pm Standard Error of Mean (SEM) of each experiment. All statistical analysis except for survival rate were accomplished by t-Test the SPSS (Statistical Packages for Social Science) version 22 software and p-value less than 0.05 was considered that there is statistically significant differences. The results of survival rate were analysed and presented with Kaplan Meier test.

\section{Results and Discussion}

Aloe Vera gel is effective in boosting both cellular and humoral immune response through attachment on mannose receptor on macrophage. Acemannan stimulate macrophage to produce inflammatory cytocins which demonstrates tumor suppressor effect (as it have been seen in our study). Wang et al. [25] evaluated the antitumor activity of aloe polysaccharides (AP) and they observed recduction in the tumor weight and found out that AP also could help to decrease chemotherapy side-effects and also improve the antitumor effects of CTX, ADM and 5FU. It is hypothesize that aloe vera polysaccharides can increased the level of IL-2 and TNF- $\alpha$ in the serum of mice and enhance immune responses to suppress tumor cell and subsequently resulted in higher survival rate and may probably has the potential to be used as a safe and nontoxic supplement in cancer patient in combination of conventional therapy [25].

\section{IFN- $\gamma$ and IL-4 Cytokines Assay}

Linna Zhang et al. study in 1996 on mouse macrophage cell line that situated in Raw 264.7 cells, indicated that Acemannan lead to stimulate cytokines production by macrophage, nitric oxide release, surface molecule expression and cell morphological changes [19]. Consequence of long-term Aloe Vera gel oral administration in this project, not only adjusts some immune system indicators, but also enhances vaccine efficacy in protection debate and improves vaccine response in some parameters. Although, this subject is much more observed in our control GEL group [26]. Kemp et al. after an experiment on the model animal, reported that $800 \mathrm{mg}$ of daily Acemannan increased number of current monocyte, macrophages and also improved clinical symptoms [27] IL-4 values increased in the GEL ALONE group and E7d-ALUM-GEL group, but it was not statistically significant ( $p>0.05)$. In this study, GEL ALONE group has the most increase in IL-4 level in comparison to other groups although this is not significant $(\mathrm{p}>0.05$ ) (Figure 2). IL-4 level in E7d-ALUM, E7d-MONT-GEL, E7d-MONT and PBS group was approximately the same. Secreted IFN- $\gamma$ by Th1, T CD8+ lymphocyte and natural killer cell, is the most important cytokine and has crucial role against tumors. Thompson et al. have proven that IFN- $\gamma$ as one of the main cytokines suppressing cancer, helped the cytotoxic properties of CD8+ [28]. The Aloe Vera mucilage introduce as a plant composition that activate the differentiation of CD4 lymphocytes via regulating the expression of Th1 and Th2 cytokines Yang et al. [29]. used several plants polysaccharide including Aloe Polysaccharide (AP) as an adjuvant to evaluate its adjuvant activity on vaccine, and they detected higher level of antibody titer, increased cytokine and T-lymphocyte proliferation in peripheral blood [30]. 


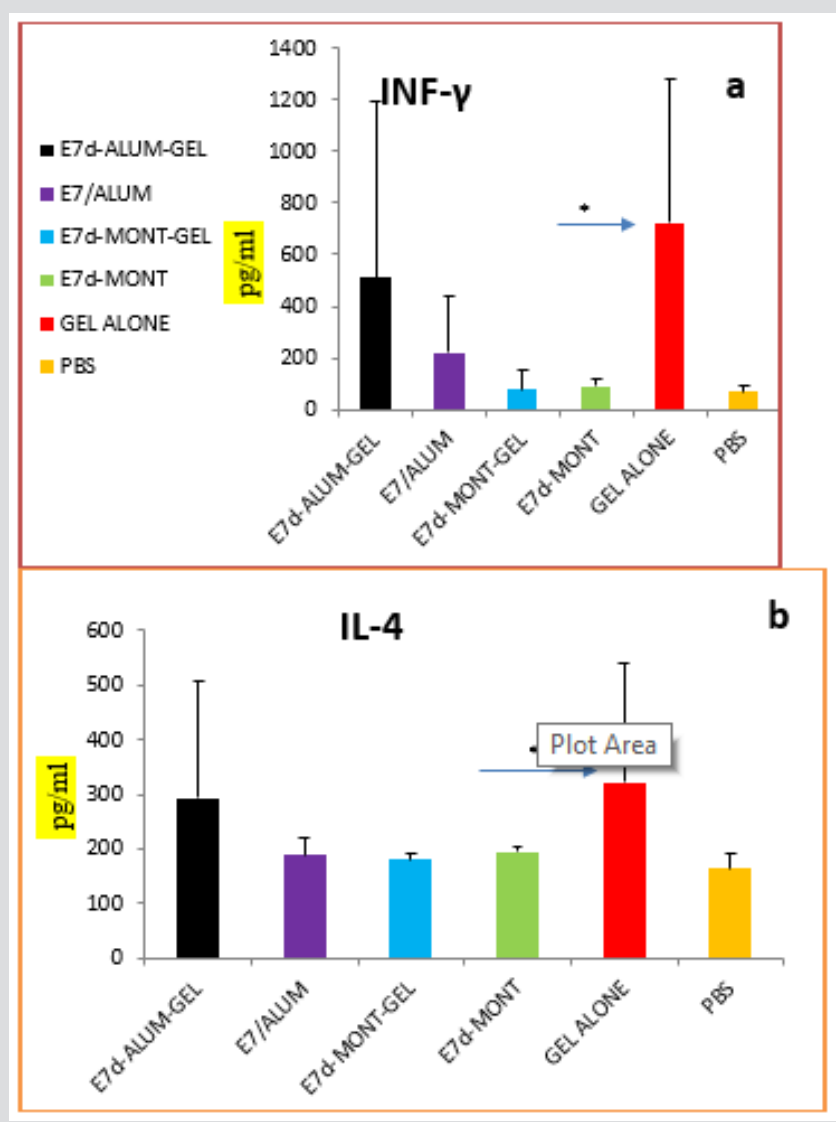

Figure 2: IFN- $\gamma$ and IL-4 cytokines and IFN- $\gamma /$ IL-4 ratio increase in both of GEL ALONE and vaccinated E7d-ALUM-GEL groups in comparison to other groups $(p>0.05)$.

It seems that reinforcing activities of Acemannan is occured through activation of macrophages to produce inflammatory cytokines such as IL-1, IL-6, TNF- $\alpha$ and IFN- $\gamma$. It is proposed that this happens by binding of Acemannan to the mannose receptors on macrophages [15,31]. In the current study, there was an increase in IFN- $\gamma$ level of GEL ALONE and E7d-ALUM-GEL group in comparison to other groups but the increase was much more considerable in GEL ALONE group than E7d-ALUM-GEL group. However the increased level of IFN- $\gamma$ cytokine in these two groups were not statistically significant $(p>0.05)$.

The enhancement of IFN- $\gamma$ in E7-ALUM-GEL group versus E7ALUM group, has showed the stimulation of immune response toward Th1 pattern. Despite our hypothesis, GEL ALONE group showed maximum amount of IFN- $\gamma$ secretion [32]. IFN- $\gamma$ level in E7d-MONT-GEL, E7d-MONT and PBS group was approximately the same. There was no significant difference in IFN- $\gamma$ cytokine level in E7d-MONT-GEL group in comparison with (PBS and E7d-MONT) test and control groups ( $p>0.05$ ) (Figure 2). Also, an increase in IFN $\gamma /$ IL-4 ratio has been detected in E7d-ALUM-GEL and GEL ALONE group in comparison to other experimental groups that support the shift of immune system in favor of cellular immunity and Th1 responses by oral administration of the Aloe Vera gel to mice that suffer from cancer, and the increase was much more significant in GEL ALONE group, although the increase was not statistically significant ( $\mathrm{p}>0.05)$.

\section{Specific Total IgG Antibodies}

Monitoring of total IgG antibody concentration demonstrates that the most increase in total antibody level belonged to E7dMONT-GEL group in comparison to all of the groups. Also, a remarkable meaningful increasement of total IgG in E7d -ALUMGEL group in camparison to GEL ALONE and PBS groups ( $p=0.03$ ) has been detected (Figure 3a).

\section{IgG Isotypes}

IgG1 and IgG2a are isotype of antibody that represent declination to Th2 and Th1 respectively. In this study the results show that IgG 1 and IgG2a have an increase in test group mice including E7dALUM-GEL, E7D-ALUM, E7d-MONT-GEL and E7d-MONT group in comparison to GEL ALONE and PBS group ( $\mathrm{p}=0.001)$. As it has been shown in the figure, no significant differences in IgG2a level were observed among the E7d-ALUM and E7d -ALUM-GEL groups ( $p>0.05$ ), but a meaningfull increase of IgG2a level was observed in E7d-MONT-GEL and E7d- MONT groups compared to other test groups $(\mathrm{p}=0.000$ ) (Figure $3 \mathrm{~b}$ ) but the most increase in IgG2a leve has been detected in E7d-MONT-GEL. In the present study IgG1 level increased in all vaccinated mice irrelevant to aloe vera gel 
and IL-4 (as IL-4 switch antibodis toward IgG1, it may interprete that IL-4 level were not high enough to stimulate IgG1 secreation). As it is known, IFN- $\gamma$ swich immune system toward Th1 and also result in the production IgG2a, but the results of present study do not show this pattern, and the increasment of IgG2a in E7d-MONTGEL and E7d-GEL group was mostly realted to the properties of Montanide as adjuvant. On the other hand, the significant increases in property of IgG2a level in E7d-MONT-GEL in comparison to E7d-
MONT group and also the significant increase of total IgG in E7dMONT-GEL group in comparison to E7d-MONT and other groups, can be concluded that aloe vera gel has this potential to be used as coadjuvant in combination with Montanide in vaccice therapy to enhance cellular and humoral immune system protection. As respected, no increase was observed in IgG1 and IgG2a (Figure 3c) level in GEL ALONE and PBS group.
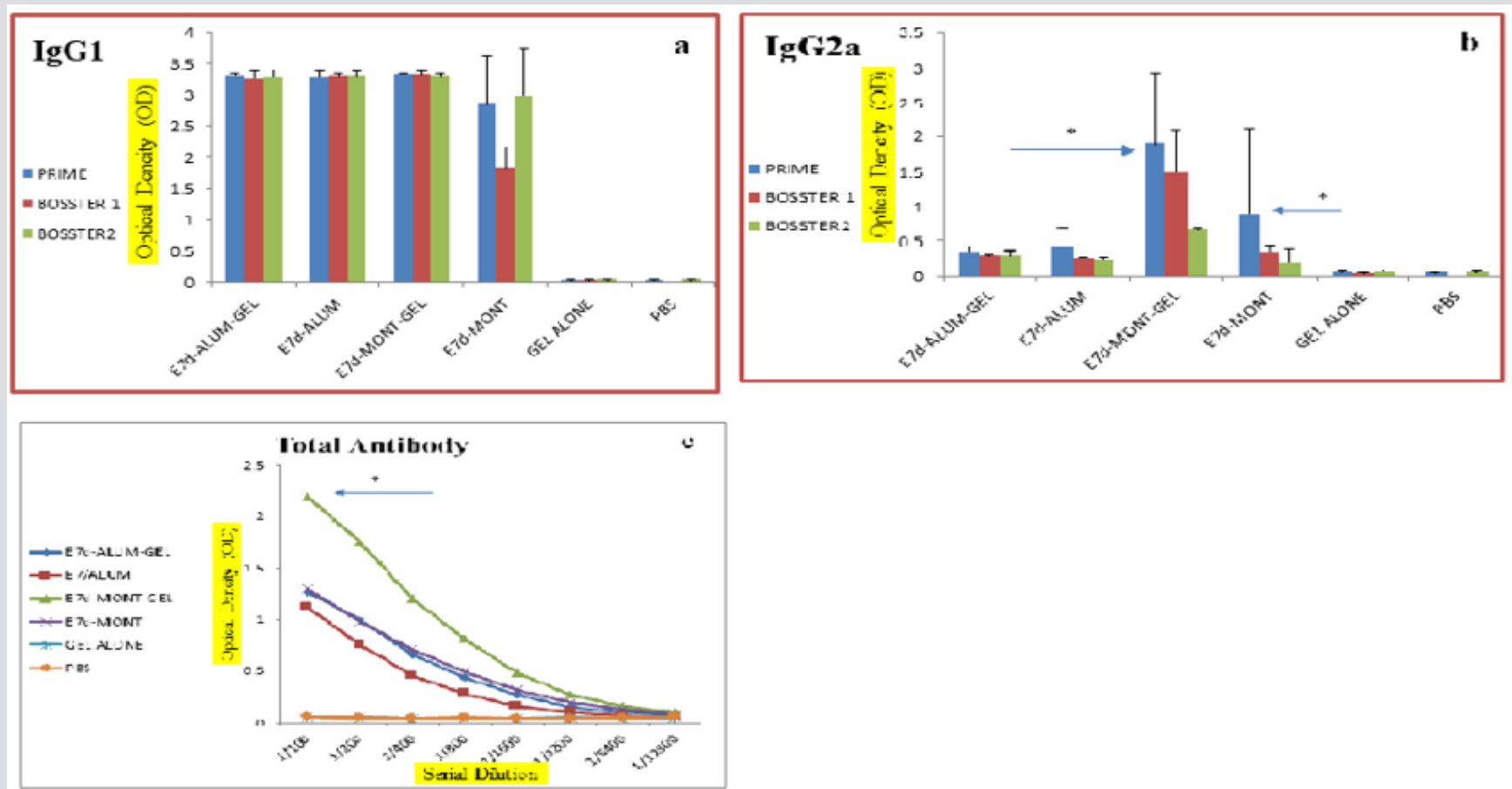

Figure 3: a) Results of IgG1 show an increase $(p=0.001)$ in all group except GEL ALONE and PBS group.b) No significant differences in IgG2a level were observed among the E7d-ALUM and E7d -ALUM-GEL groups ( $\mathrm{p}>0.05$ ), but a meaningfull increase of IgG2a level was observed in E7d-MONT-GEL and E7d-MONT groups compared to other test groups ( $\mathrm{p}=0.001$ ). c) The most belonged to E7d-MONT-GEL group in comparison to E7d-MONT and other groups. Also, a remarkable meaningful increasement of total IgG in E7d -ALUM-GEL group in camparison to GEL ALONE and PBS groups $(p=0.03)$ has been detected.

\section{Tumor Volume Changes}

Tumor volume $\left(\mathrm{mm}^{3}\right)$ changes showed the lowest ratio of tumor growth rate and the most reduction of proportion of tumor growth in the Aloe Vera Gel group, mostly in GEL ALONE group, but the data of tumor growth was not statistically significant (Figure 4a). Groups that have Alum as adjuvant show better result in proportion of tumor size compared to groups that have Montanide as adjuvant that may represent the much better response of Alum as adjuvant in this study but none of this group show reduction of tumor size as effective as group that only received Aloe Vera gel and this may be due to immunostimulating and antitumor activity effect of Aloe Vera polysaccharide (Figure 4b \& 4c). According to the research was done by Reynolds et al. in 2004, Acemannan is known as an in vivo and in vitro immunomodulatory polysaccharide, so when it was administered to mice, acemanan induced complet treatment or reduction of tumors volume in mice [20].

\section{Survival Rate}

Based on the results of this study, highest survival rates was belonged to E7d-ALUM-GEL and GEL ALONE groups (Figure 5). In addition, in 1991 Harris et al. reported administration of Acemannan has shown increased tumor necrosis and prolonged survival rate. Acemannan has shown an activity as an immunestimulatory supplement against the virus and boosts survival rate in animals infected with the virus which has also been demonstrated in our study according to the reduction in growth rate of tumor size and increased survival [15]. 


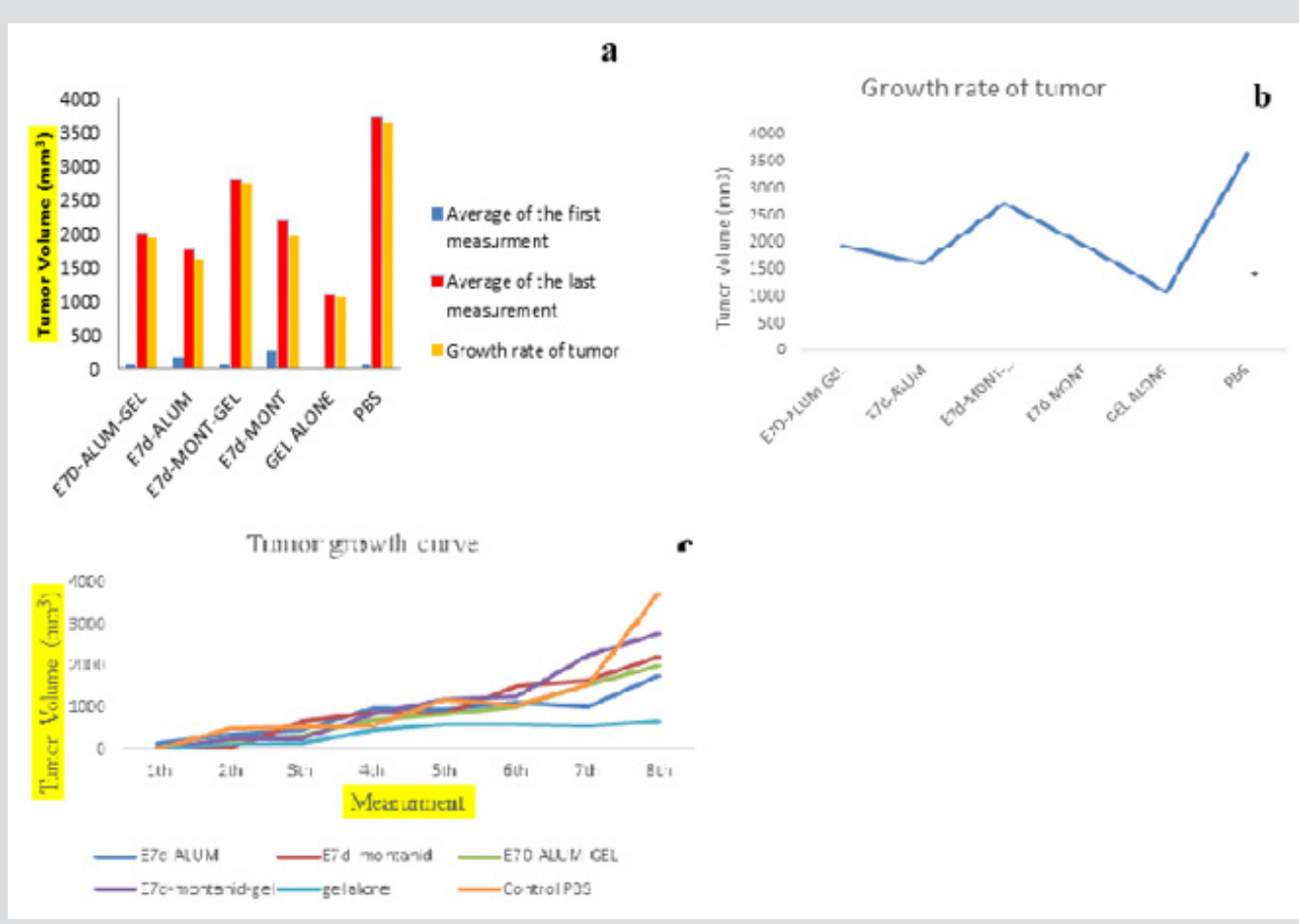

Figure 4: a) the average of the last measurement and tumor growth rate is minimum in GEL ALONE group. b and c) the lowest tumor growth rate and the greatest reduction of proportion of tumor growth in GEL ALONE group that only received Aloe Vera gel.

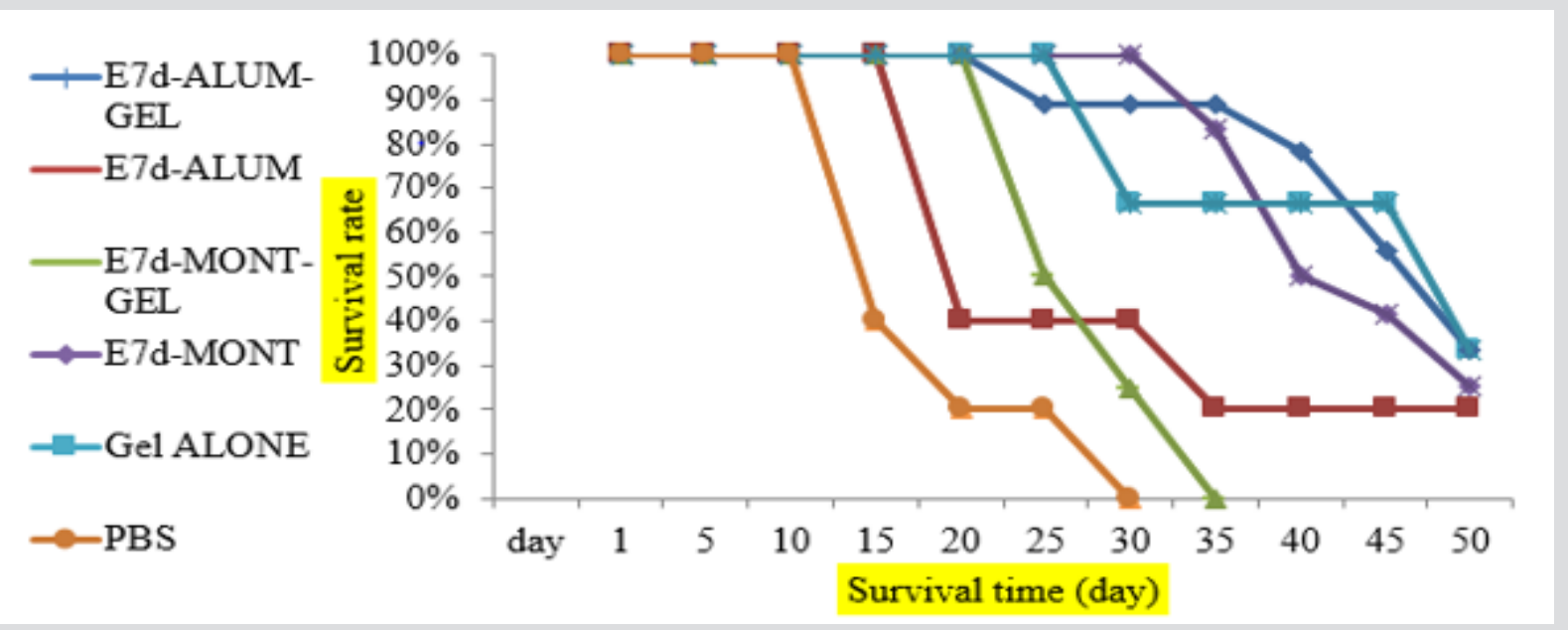

Figure 5: The rate of deth in GEL ALONE and E7d- ALUM - GEL groups is minimum and the mice in both of this groups persist against cancer. The results of survival rate were analyzed and presented with Kaplan Meier test.s

\section{Conclusion}

To sum up with the results of present work and also what has been known about APs it can be concluded that this components of Aloe vera has the potential of enhancing the immune responses and may have the capacity to be used as a supplement to help the current protocol of cancer treatment. This effect was possibly derived from inducing IL-2, TNF- $\alpha$, IFN- $\gamma$ and other cytokines which are produced in the body and also improves the innate immune response activity
[25]. Aloe Vera extracted polysaccharides could improve cellular and humoral immune responses and can reinforced vaccine immunization although this effect was not observed in separated administration model in our work and it would be better to study the effect of Aloe Vera extracted gel polysaccharides in the formulation of vaccine instead of seprated administration. It is likely that this natural polysaccharide has impact on the stimulation of the immune responses and may be considered as a beneficial supplement in combination with chemotherapy drugs during cancer therapy. 


\section{Conflict of Interest}

The authors declare that they have no conflict of interest.

\section{Ethical Approval}

In this study all experimental procedures using animals was carried out according to the Animal Care and Use Guidelines of Pasteur Institute of Iran. (Tehran, Iran).

\section{Acknowledgment}

This work was supported by Deputy of Research of Tehran University of Medical Sciences [grant number 34249] Iran National Science Foundation (INSF), [grant number 94007096区.

\section{References}

1. Jemal A, Bray F, Center MM, Ferlay J, Ward E, et al. (2011) Global cancer statistics. CA: a cancer journal for clinicians 61(2): 69-90.

2. Bahrami AA, Ghaemi A, Tabarraei A, Sajadian A, Gorji A, et al. (2014) DNA vaccine encoding HPV-16 E7 with mutation in L-Y-C-Y-E pRbbinding motif induces potent anti-tumor responses in mice. Journal of Virological Methods 206: 12-18.

3. Chen J (2015) Signaling pathways in HPV-associated cancers and therapeutic implications. Reviews in medical virology 25(1): 24-53.

4. Zur Hausen H (2009) The Search for Infectious Causes of Human Cancers: Where and Why (Nobel Lecture). Angewandte Chemie International Edition 48(32): 5798-808.

5. Zandberg DP, Bhargava R, Badin S, Cullen KJ (2013) The role of human papillomavirus in nongenital cancers. CA: a cancer journal for clinicians 63(1): 57-81

6. Zaravinos A (2014) An updated overview of HPV-associated head and neck carcinomas. Oncotarget 5(12): 3956-3969.

7. Nicol AF, Andrade CV, Russomano FB, Rodrigues LLS, Oliveira NS, et al. (2016) HPV vaccines: a controversial issue? Brazilian Journal of Medical and Biological Research 49(5).

8. Monsonego J (2006) Cervical cancer prevention: the impact of HPV vaccination.Gynecologie, obstetrique \& fertilite 34(3): 189-201.

9. Gonçalves AK, Cobucci RN, Rodrigues HM, De Melo AG, Giraldo PC (2014) Safety, tolerability and side effects of human papillomavirus vaccines: a systematic quantitative review. The Brazilian Journal of Infectious Diseases 18(6): 651-659.

10. Petrone L, Ammendolia MG, Cesolini A, Caimi S, Superti F, et al. (2011) Recombinant HPV16 E7 assembledinto particles induces an immune response and specific tumour protection administered without adjuvant in an animal model. Journal of Translational Medicine 9: 69.

11. Hellner K, Münger K (2011) Human papillomaviruses as therapeutic targets in human cancer. Journal of clinical oncology 29(13):1785-1794.

12. Van Der Burg SH, Melief CJ (2011) Therapeutic vaccination against human papilloma virus induced malignancies. Current opinion in immunology 23(2): 252-257.

13. Van Damme P, Leroux Roels G, Simon P, Foidart JM, Donders G, et al. (2014) Effects of varying antigens and adjuvant systems on the immunogenicity and safety of investigational tetravalent human oncogenic papillomavirus vaccines: Results from two randomized trials. Vaccine 32(29): 3694-3705.

14. Petrovsky N, Aguilar JC (2004) Vaccine adjuvants: Current state and future trends. Immunol Cell Biol 82(5): 488-496.
15. Petrovsky N, Aguilar JC (2004) Vaccine adjuvants: current state and future trends. Immunology and cell biology 82(5): 488-496.

16. Coffman RL, Sher A, Seder RA (2010) Vaccine adjuvants: putting innate immunity to work. Immunity 33(4): 492-503.

17. Mahdavi M, Ebtekar M, Azadmanesh K, Mahboudi F, Khorramkhorshid H, et al. (2010) HIV-1 Gag p24-Nef fusion peptide induces cellular and humoral immune response in a mouse model. Acta virologica 54(2): 131.

18. Harlev E, Nevo E, Lansky EP, Ofir R, Bishayee A (2012) Anticancer Potential of Aloes: Antioxidant, Antiproliferative, and Immunostimulatory Attributes. Planta Med 78(09): 843-852.

19. Zhang L, Tizard IR (1996) Activation of a mouse macrophage cell line by acemannan: themajor carbohydrate fraction from Aloe vera gel. Immunopharmacology 35(2): 119-128.

20. Reynolds T (2004) Aloes: the genus Aloe: CRC press, Florida, United States.

21. Chokboribal J, Tachaboonyakiat W, Sangvanich P, Ruangpornvisuti V, Jettanacheawchankit S, et al. (2015) Deacetylation affects the physical properties and bioactivity of acemannan, an extracted polysaccharide from Aloe vera. Carbohydrate polymers 133: 556-566.

22. Keithley JK, Swanson B, Mikolaitis SL, DeMeo M, Zeller JM, et al. (2013) Safety and Efficacy of Glucomannan for Weight Loss in Overweight and Moderately Obese Adults. Journal of Obesity: 610908.

23. Zhan X, Jia L, Niu Y, Qi H, Chen X, et al. (2014) Targeted depletion of tumour-associated macrophages by an alendronate-glucomannan conjugate for cancer immunotherapy. Biomaterials 35(38): 1004610057.

24. Songsiripradubboon S, Banlunara W, Sangvanich P, Trairatvorakul C, Thunyakitpisal P (2015) Clinical, radiographic, and histologic analysis of the effects of acemannan used in direct pulp capping of human primary teeth: short-term outcomes. Odontology 104(3): 329-337.

25. Wang Z, Wang Y, Huang Z, Zhong S, Wu Y, et al. (2001) Study on antitumor effect and mechanism of aloe polysaccharides]. Zhong Yao Cai 24(5): 350-353.

26. Harris C, Pierce K, King G, Yates K, Hall J, et al. (1991) Efficacy of acemannan in treatment of canine and feline spontaneous neoplasms. Molecular biotherapy 3(4): 207-213

27. Kemp M, Kahlon J, Chinnah A, Carpenter R, Mc Analley B, et al. (1990) Invitro evaluation of the antiviral effects of acemannan on the replication and pathogenesis of HIV-1 and other enveloped viruses: Modification of the processing of glycoprotein precursors. Antiviral Res 13(1): 83

28. Thompson ED, Enriquez HL, Fu YX, Engelhard VH (2010) Tumor masses support naive $\mathrm{T}$ cell infiltration, activation, and differentiation into effectors. Journal of Experimental Medicine 207(8): 1791-1804.

29. Yu H, Dong Z, Yang Z (2002) Molecular biological study of Aloe vera in the treatment of experimental allergic rhinitis in rat. Journal of clinical otorhinolaryngology 16(5): 229-231.

30. Yang Y, Wei K, Yang S, Li B, Zhang Y, et al. (2015) Co-adjuvant effects of plant polysaccharide and propolis on chickens inoculated with Bordetella avium inactivated vaccine. Avian Pathology 44(4): 248-253.

31. Snapper CM, Finkelman FD, Paul WE (1988) Regulation of IgG1 and IgE production by interleukin 4. Immunological reviews 102(1): 51-75.

32. Montañez MI, Ruiz Sanchez AJ, Perez Inestrosa E (2010) A perspective of nanotechnology in hypersensitivity reactions including drug allergy. Current opinion inallergy and clinical immunology 10(4): 297-302. 
ISSN: 2574-1241

DOI: $10.26717 /$ BJSTR.2019.16.002873

Mohammad Hossein Y, Mehdi M. Biomed J Sci \& Tech Res

(c) This work is licensed under Creative

Submission Link: https://biomedres.us/submit-manuscript.php

\begin{tabular}{ll} 
BIOMEDICAL & \multicolumn{1}{c}{ Assets of Publishing with us } \\
RESEARCHES & - Global archiving of articles \\
& - Immediate, unrestricted online access \\
& - Rigorous Peer Review Process \\
\end{tabular}

